\title{
UMIDADE DE EQUILÍBRIO DE PAINÉIS DE MADEIRA ${ }^{1}$
}

\author{
Graciane Angélica da Silva ${ }^{2}$, Lourival Marin Mendes ${ }^{3}$, Paulo Fernando Trugilho ${ }^{3}$, Fábio Akira Mori ${ }^{3}$ e
} Izaías Fernandes dos Santos ${ }^{4}$

\begin{abstract}
RESUMO - Este estudo teve como objetivo a determinação da umidade de equilíbrio de painéis de madeira numa câmara de climatização e comparação dos seus valores com os obtidos através da equação de Nelson. O trabalho foi desenvolvido na Unidade Experimental de Produção de Painéis de Madeira da Universidade Federal de Lavras. Foram utilizadas amostras de aglomerado, compensado multilaminado e sarrafeado, chapa dura, OSB (Oriented Strand Board), MDF (Medium Density Fiberboard), HDF (High Density Fiberboard), madeiracimento e madeira-plástico, sendo esses produtos de escala industrial e experimental. As dimensões dos corposde-prova foram de $2,60 \mathrm{~cm}$ de largura por $2,60 \mathrm{~cm}$ de comprimento. As espessuras foram aquelas mais utilizadas comercialmente de cada produto, sendo utilizadas cinco repetições por painel. Os corpos-de-prova foram pesados e levados para uma câmara de climatização, onde foram submetidos a diferentes condições de umidade relativa $(90,80,70,60,50$ e $40 \%)$ e uma temperatura constante de $30^{\circ} \mathrm{C}$. Determinaram-se a histerese e a umidade de equilíbrio, que variaram de 40 a $90 \%$. Os resultados indicaram que a equação de Nelson se mostrou eficiente na estimativa da umidade de equilíbrio e que não existe em média diferença de histerese entre os produtos estudados, mostrando que todos apresentam a mesma estabilidade dimensional. Observou-se, também, que o revestimento dos painéis não afetou a umidade de equilíbrio.
\end{abstract}

Palavras-chave: Umidade de equilíbrio, isotermas de sorção, equação de Nelson e painéis de madeira.

\section{EQUILIBRIUM MOISTURE CONTENT OF WOOD PANELS}

\begin{abstract}
This study aimed at determining the equilibrium moisture content of wood panels in a climatization chamber and comparing the values of equilibrium moisture content found with those given by Nelson's equation. The research was developed at the Experimental Unit of Wood Panel Production of the Universidade Federal de Lavras. Samples of particleboard, plywood, hardboard, OSB (Oriented Strand Board), MDF (Medium Density Fiberboard), HDF (High Density Fiberboard), cement wood and plastic wood were taken both from commercial wood and from experiments and trials. The specimen dimensions were $2.60 \mathrm{~cm}$ width by 2.60 $\mathrm{cm}$ length, and thickness commercially used of each product. Five repetitions were carried out for each panel. The specimens were weighted and then taken to the climatization chamber under different conditions of relative humidity $(90,80,70,60,50$ and $40 \%)$ at $30^{\circ} \mathrm{C}$. The hysteresis and equilibrium moisture content ranged from 40 to $90 \%$. The results indicate that Nelson's equation is efficient for estimating the equilibrium moisture content and that there is no hysteresis difference in the studied products. All of them presented the same dimensional stability. It was also observed that panel coating did not affect the equilibrium moisture content.
\end{abstract}

Keywords: Equilibrium moisture content, isotherms, sorption Nelson's equation and wood panels.

\footnotetext{
${ }^{1}$ Recebido em $1^{\circ} .06 .2004$ e aceito para publicação em 20.04.2005.

${ }^{2}$ Mestre em Ciências Florestais. Rua Nelo Totti, 183, Centro, 37405-000 Monsenhor Paulo-MG. E-mail: <gracianeas@ yahoo.com.br>.

${ }_{3}^{3}$ Departamento de Ciências Florestais da UFLA, Cx. Postal 37,37200-000 Lavras-MG. E-mail: <lourival@ ufla.br>e <trugilho@ufla.br>. <morif@ufla.br>.

${ }^{4}$ Estudante de Engenharia Florestal da Universidade Federal de Lavras (UFLA). E-mail: <ifsantos@ eflorestal.ufla.br>.
} 


\section{INTRODUÇÃO}

Segundo Suchsland (1972) o conhecimento da relação da umidade de equilíbrio com a umidade relativa (UR) e temperatura $\left({ }^{\circ} \mathrm{C}\right)$ é uma ferramenta importante para a utilização de produtos compostos de madeira. Essa relação é conhecida como isoterma de sorção e afeta, de forma bastante significativa, a resistência mecânica e a estabilidade dimensional de diferentes produtos. Para determinação dessa isoterma de sorção é necessária uma análise detalhada dos problemas relacionados com a umidade do material, como empenamentos na manufatura do painel e inchamento e contração na estrutura do composto (BURCH et al., 1992; WU e SUCHSLAND, 1996).

Os compostos reconstituídos de madeira apresentam isotermas de sorção essencialmente diferentes daquelas apresentadas pela madeira maciça (SUCHSLAND, 1972; WU e SUCHSLAND, 1996). Esse fato é atribuído às altas temperaturas utilizadas, durante a prensagem, no processo de manufatura (SUCHSLAND, 1972). Alguns estudos experimentais foram conduzidos com o objetivo de determinar a isoterma de sorção de diferentes produtos compostos de madeira (HEEBINK e HASKELL, 1962; BURCH et al.,1992). Nenhum desses estudos, no entanto, envolveu uma análise mais aprofundada com dados de regressão para ajuste de modelos.

As isotermas de sorção variam de espécie para espécie, e essas diferenças são atribuídas às diferentes proporções, em que os constituintes (celulose, polioses e lignina) entram para formar a madeira das diferentes espécies e as possíveis inter-relações entre eles, nas paredes celulares (GALVÃO, 1985). As polioses têm a maior capacidade sortiva, ou seja, em torno de $47 \%$, seguida pela celulose com $37 \%$ e a lignina com $16 \%$ (MESQUITA, 2000).

Nelson (1983) desenvolveu uma equação baseada no modelo da energia livre de Gibbs e determinou, através de análise de regressão, os coeficientes originais dessa equação para alguns produtos à base de madeira.

Os parâmetros que definem a isoterma de sorção variam com o processo de sorção e o tipo de produto. A determinação desses parâmetros torna possível utilizar o Modelo Analítico de Nelson, como uma ferramenta, para prever, por exemplo, umidade que flui através das paredes de edifícios (BURCH et al., 1992) e o gradiente de umidade em painéis de revestimento de mobílias (WU e SUCHSLAND, 1996).

\section{R. Árvore, Viçosa-MG, v.29, n.4, p.639-646, 2005}

A teoria de Nelson (1983) e Wu (1999) é um modelo desenvolvido para estimar a umidade de equilíbrio de produtos compostos de madeira, por exemplo: MDF, HPL, OSB e chapa dura. No Brasil, não se tem relato da utilização dessa equação ou de outras para estimar a umidade de equilíbrio de compostos de madeira.

Este trabalho teve como finalidade avaliar a umidade de equilíbrio em diferentes condições de umidade relativa $(90,80,70,60,50$ e $40 \%)$, a uma temperatura de $30^{\circ} \mathrm{C}$, dos diversos painéis produzidos no Brasil, com espessuras diferentes e com e sem revestimentos. Também, objetivaram-se comparar os valores de umidade de equilíbrio calculados pela equação de Nelson com os observados na câmara de climatização.

\section{MATERIAL E MÉTODOS}

O trabalho foi desenvolvido na Unidade Experimental de Produção de Painéis de Madeira do Departamento de Ciências Florestais da Universidade Federal de Lavras. Utilizaram-se amostras de aglomerado, compensado multilaminado e sarrafeado, chapa dura, OSB, MDF, HDF, madeira-cimento e madeira-plástico, sendo a maioria desses painéis de produção industrial; os dois últimos foram produzidos em laboratório.

As dimensões dos corpos-de-prova foram de $2,60 \mathrm{~cm}$ de largura por $2,60 \mathrm{~cm}$ de comprimento, segundo a metodologia desenvolvida por Wu (1999). As espessuras foram aquelas mais empregadas comercialmente de cada produto, sendo utilizadas cinco repetições por painel, em um delineamento inteiramente casualizado (Tabela 1).

As amostras foram pesadas numa balança com carga máxima de 2.000 g, carga mínima de 0,5 g e levadas para uma câmara de climatização, que trabalha com uma variação de temperatura de 10 a $100{ }^{\circ} \mathrm{C}$ e umidade relativa variando na faixa de 30 a $95 \%$. As amostras foram submetidas a diferentes condições de umidade relativa para uma temperatura constante de $30^{\circ} \mathrm{C}$. As condições iniciais de umidade relativa foram de 90 , $80,70,60,50$ e $40 \%$, para se obter a primeira curva de dessorção. Em seguida, as amostras foram expostas à umidade relativa crescente sob as mesmas condições, para se obter a curva de sorção. Repetiu-se, então, o primeiro procedimento para obtenção da segunda curva de dessorção. Ao final, as amostras foram levadas à estufa com temperatura em torno de $103^{\circ} \mathrm{C}$ até peso constante, quando foram pesadas novamente para a determinação da umidade. 
Tabela 1 - Médias das espessuras e revestimentos de cada produto Table 1-Means of thickness and coating of each product

\begin{tabular}{|c|c|}
\hline Tratamento & Descrição do Tipo de Revestimento por Tratamento de Acordo com ABIPA (2000) \\
\hline & Compensado \\
\hline 1 & $\begin{array}{l}\text { Compensado multilaminado, com espessura de } 18,15 \mathrm{~mm} \text { e revestido com lâmina decorativa natural e em } \\
\text { uma das faces revestida com PVC }\end{array}$ \\
\hline 2 & $\begin{array}{l}\text { Compensado sarrafeado, com espessura de } 19,09 \mathrm{~mm} \text { e revestido com lâmina decorativa natural em ambas } \\
\text { as faces }\end{array}$ \\
\hline 3 & Compensado multilaminado, com espessura de $16,98 \mathrm{~mm}$ sem revestimento \\
\hline 4 & $\begin{array}{l}\text { Compensado multilaminado, com espessura de } 15,17 \mathrm{~mm} \text { revestido em ambas as faces com lâminas decorativas } \\
\text { naturais }\end{array}$ \\
\hline 5 & $\begin{array}{l}\text { Compensado sarrafeado, com espessura de } 18,89 \mathrm{~mm} \text {, revestido em ambas as faces com lâminas decorativas } \\
\text { naturais }\end{array}$ \\
\hline & Aglomerado \\
\hline 6 & Aglomerado, com espessura de $15,87 \mathrm{~mm}$, revestido com lâmina decorativa natural em ambas as faces \\
\hline 7 & Aglomerado, com espessura de $16,21 \mathrm{~mm}$, revestido com lâmina decorativa natural em ambas as faces \\
\hline 8 & Aglomerado, com espessura de $15,24 \mathrm{~mm}$, revestido com laminado Baixa Pressão (BP) em ambas as faces \\
\hline 9 & Aglomerado, com espessura de $10,56 \mathrm{~mm}$, revestido com Finish Foil em ambas as faces \\
\hline 10 & Aglomerado, com espessura de $15,14 \mathrm{~mm}$, sem revestimento em ambas as faces \\
\hline 11 & $\begin{array}{c}\text { Aglomerado, com espessura de } 3,36 \mathrm{~mm} \text {, sem revestimento em ambas as faces } \\
\text { Chapa Dura }\end{array}$ \\
\hline 12 & Chapa dura, com espessura de $3,11 \mathrm{~mm}$, revestido com finish foil em uma das faces \\
\hline 13 & Chapa dura, com espessura de $3,12 \mathrm{~mm}$, revestido com laminado baixa pressão (BP) em uma das faces \\
\hline 14 & $\begin{array}{c}\text { Chapa dura, com espessura de } 3,15 \mathrm{~mm} \text { sem revestimento em ambas as faces } \\
\text { MDF }\end{array}$ \\
\hline 15 & MDF, com espessura de $15,39 \mathrm{~mm}$, sem revestimento em ambas as faces \\
\hline 16 & MDF, com espessura de $15,27 \mathrm{~mm}$, revestido com finish foil em ambas as faces \\
\hline 17 & $\begin{array}{c}\text { MDF, com espessura de } 3,16 \mathrm{~mm} \text {, sem revestimento em ambas as faces } \\
\text { OSB }\end{array}$ \\
\hline 18 & OSB, com espessura de $9,46 \mathrm{~mm}$, sem revestimento em ambas as faces \\
\hline 19 & OSB, com espessura de $16,07 \mathrm{~mm}$, revestido com lâmina decorativa natural \\
\hline 20 & $\begin{array}{c}\text { OSB, com espessura de } 18,48 \mathrm{~mm} \text {, sem revestimento em ambas as faces } \\
\text { Madeira-Cimento }\end{array}$ \\
\hline 21 & Madeira cimento, com espessura de $15,92 \mathrm{~mm}$, sem revestimento em ambas as faces \\
\hline 22 & $\begin{array}{c}\text { Madeira cimento, com espessura de } 15,76 \mathrm{~mm} \text {, sem revestimento em ambas as faces } \\
\text { Madeira Plástico }\end{array}$ \\
\hline 23 & $\begin{array}{l}\text { Madeira plástico, com espessura de } 12,41 \mathrm{~mm} \text {, sem revestimento em ambas as faces } \\
\text { HDF }\end{array}$ \\
\hline 24 & HDF, com espessura de $6,13 \mathrm{~mm}$, sem revestimento em ambas as faces \\
\hline
\end{tabular}

Determinou-se a histerese, através da razão entre as umidades de equilíbrio da madeira, obtidas na curva de adsorção e dessorção (A/D), em que UE2 corresponde à curva de sorção e UE1 e UE3, às curvas da primeira e segunda dessorções, respectivamente.

Em cada condição de umidade relativa, o peso das amostras foi monitorado, com o objetivo de verificar o momento em que se estabelecesse o equilíbrio. As amostras de madeira submetidas a pesagens subsequientes, realizadas em intervalos de 24 horas, cuja variação foi inferior a $1 \%$, foram consideradas em condição de completa umidade de equilíbrio.
A equação utilizada foi desenvolvida por Nelson (1983) e ajustada por Wu (1999), com base no modelo da energia livre de Gibbs. Essa equação apresenta a seguinte estruturação:

$$
U E H=M v\left\{1,0-\frac{1}{A} \ln \left[\left(\frac{R^{*} T}{W w}\right) \ln \left(\frac{R H}{100}\right)\right]\right\}
$$

em que:

$\mathrm{UEH}=$ umidade de equilíbrio higroscópico (\%);

$\mathrm{RH}=$ umidade relativa $(\%)$;

$\mathrm{W} \mathrm{w}=$ peso molecular da água $(18 \mathrm{~g} / \mathrm{mol})$;

R. Árvore, Viçosa-MG, v.29, n.4, p.639-646, 2005 
$\mathrm{R}=$ constante universal dos gases $(1,9858 \mathrm{cal} /$ $\mathrm{mol} / \mathrm{oK})$;

$\mathrm{T}=$ temperatura absoluta $\left({ }^{\circ} \mathrm{K}\right) ;$

$\mathrm{A}=$ logaritmo natural da energia livre de Gibbs por grama de água absorvida com a umidade relativa próximo de zero; e

$\mathrm{Mv}=$ constante do material aproximado (PSF) para desorção, em porcentagem.

A equação de Nelson (1983) foi ajustada para aglomerado, OSB, chapa dura e MDF e, em seguida, feita a comparação dos valores de umidade de equilíbrio, calculada por essa mesma equação e observada na câmara de climatização.

\section{RESULTADOS E DISCUSSÃO}

Na Tabela 2, apresenta-se o resumo da análise de variância da UE1, UE2 e UE3, verificando-se também, nessa tabela, que não existe diferença significativa de UE1, UE2 e UE3, entre os tratamentos.

$\mathrm{Na}$ Tabela 3 estão apresentados os resultados médios das umidades de equilíbrio na temperatura de $30{ }^{\circ} \mathrm{C}$ e umidades relativas de 40, 50, 60, 70, 80 e 90\%, observadas na câmara de climatização, bem como os respectivos coeficientes de histerese. Não existe diferença significativa dos coeficientes de histerese por tratamento e entre produtos. Pelos resultados, observa-se que existe pequena variação na umidade de equilíbrio entre os tratamentos avaliados, principalmente nas condições de umidade relativa mais alta.

De modo geral, os coeficientes de histerese foram maiores nas umidades de equilíbrio mais baixas. Os valores médios de histerese, no compensado, variaram

Tabela 2 - Resumo da análise de variância de UE1, UE2 e UE3

Table 2 - Summary of the analysis of variance for UEI, UE2 and UE3

\begin{tabular}{lcccc}
\hline \multirow{2}{*}{ FV } & GL & \multicolumn{3}{c}{ QM } \\
\cline { 3 - 5 } & & UE1 & UE2 & UE3 \\
\hline Tratamento & 23 & $13,9878 \mathrm{NS}$ & $11,8376 \mathrm{NS}$ & $11,8765 \mathrm{NS}$ \\
Erro & 120 & 11,4672 & 10,6058 & 10,5086 \\
Total & 143 & & & \\
\hline
\end{tabular}

NS = não-significativos.

$\mathrm{NS}=$ not significant

R. Árvore, Viçosa-MG, v.29, n.4, p.639-646, 2005 de 0,91 a 0,93, sendo a média geral de 0,92. No aglomerado, os valores médios variam de 0,90 a 0,96 , sendo a média geral de 0,92; o valor médio de histerese de 0,96 foi encontrado no tratamento 9 (aglomerado com espessura de $10,56 \mathrm{~mm}$, revestido com "finish foil" em ambas as faces) em razão, possivelmente, do tipo de revestimento, em comparação com os demais tratamentos.

A chapa dura apresentou valores médios de 0,88 a 0,89 , com uma média geral em torno de 0,88 . No MDF, os valores médios variaram de 0,93 a 0,96 , caso em que a média geral foi de 0,94 . O valor médio de 0,96 foi encontrado no tratamento 17 (MDF com espessura de 3,16 $\mathrm{mm}$ sem revestimento, em ambas as faces), podendo, assim, ser devido à sua menor espessura, em comparação com os demais tratamentos. No OSB, os valores médios variaram de 0,90 a 0,92 , com uma média geral de 0,91. Na madeira-cimento, os valores médios foram compreendidos entre 0,89 e 0,95 e a média geral, em torno de 0,92, sendo, possivelmente, essa variação devida às diferentes espessuras e granulometrias entre os tratamentos.

Finalmente, na madeira-plástico e no HDF, nos quais se adotou apenas um tratamento no dispositivo experimental, as médias foram 0,90 e 0,93 , respectivamente.

Nas Tabelas 4 e 5, apresenta-se o resumo das análises de variância da diferença e umidade de equilíbrio calculada em relação à observada, respectivamente. Pode-se notar nessas tabelas que, apesar de ter ocorrido significância das médias das diferenças entre as umidades calculada e observada. Com relação à comparação entre os valores de umidade de equilíbrio calculada e observada, não se constatou diferença significativa. Tal constatação evidencia que essas unidades são iguais estatisticamente, indicando, assim, a eficiência da equação de Nelson.

Na Tabela 6, apresentam-se os valores médios de umidade de equilíbrio calculados e observados na temperatura de $30{ }^{\circ} \mathrm{C}$ e umidades relativas de 40,50 , $60,70,80$ e $90 \%$. Pode-se constatar que a equação de Nelson (1983) subestima os valores de umidades de equilíbrio. Esses valores, no entanto, são bem próximos daqueles observados na câmara de climatização, indicando, assim, que essa equação é eficiente na estimativa do cálculo da umidade de equilíbrio (\%). A maior diferença entre os valores calculados e observados foi de 2,26 , encontrada na chapa dura, na UR de $40 \%$, sendo a menor diferença $(0,01)$, encontrada no OSB, na UR de $80 \%$.

R. Árvore, Viçosa-MG, v.29, n.4, p.639-646, 2005 
Tabela 3 - Valores médios das umidades de equilíbrio na temperatura de $30{ }^{\circ} \mathrm{C}$ e coeficiente de histerese

Table 3-Average values of the equilibrium moisture content at $30{ }^{\circ} \mathrm{C}$ and coefficient of hysteresis

\begin{tabular}{|c|c|c|c|c|c|}
\hline \multicolumn{6}{|c|}{ Compensado } \\
\hline TRAT & UR & UE1 & UE2 & UE3 & HISTERESE \\
\hline 1 & 40 & 8,25 & 8,25 & 8,10 & - \\
\hline 1 & 50 & 9,58 & 9,02 & 9,83 & 0,92 \\
\hline 1 & 60 & 11,23 & 10,31 & 11,09 & 0,93 \\
\hline 1 & 70 & 13,60 & 11,42 & 12,85 & 0,89 \\
\hline 1 & 80 & 15,62 & 13,89 & 15,10 & 0,92 \\
\hline 1 & 90 & 18,87 & 18,04 & 18,04 & - \\
\hline Média* & & $12,86 \mathrm{a}$ & $11,82 \mathrm{~b}$ & $12,50 \mathrm{c}$ & $0,91 \mathrm{~d}$ \\
\hline 2 & 40 & 8,57 & 8,57 & 8,55 & - \\
\hline 2 & 50 & 10,12 & 9,56 & 10,28 & 0,93 \\
\hline 2 & 60 & 11,67 & 10,63 & 11,77 & 0,90 \\
\hline 2 & 70 & 13,60 & 12,03 & 13,49 & 0,89 \\
\hline 2 & 80 & 15,51 & 14,34 & 15,73 & 0,91 \\
\hline 2 & 90 & 17,18 & 18,45 & 18,45 & - \\
\hline Média & & $12,78 \mathrm{a}$ & $12,26 \mathrm{~b}$ & $13,05 \mathrm{c}$ & $0,91 \mathrm{~d}$ \\
\hline 3 & 40 & 9,02 & 9,02 & 8,70 & - \\
\hline 3 & 50 & 10,48 & 9,95 & 10,67 & 0,93 \\
\hline 3 & 60 & 12,10 & 11,18 & 12,08 & 0,93 \\
\hline 3 & 70 & 14,02 & 12,46 & 14,24 & 0,88 \\
\hline 3 & 80 & 17,06 & 15,36 & 17,15 & 0,90 \\
\hline 3 & 90 & 21,25 & 20,85 & 20,85 & - \\
\hline Média & & $13,99 \mathrm{a}$ & $13,14 \mathrm{~b}$ & $13,95 \mathrm{c}$ & $0,91 \mathrm{~d}$ \\
\hline 4 & 40 & 8,81 & 8,81 & 8,87 & - \\
\hline 4 & 50 & 10,34 & 9,65 & 10,39 & 0,93 \\
\hline 4 & 60 & 11,59 & 10,90 & 11,68 & 0,93 \\
\hline 4 & 70 & 12,93 & 12,14 & 13,57 & 0,89 \\
\hline 4 & 80 & 15,46 & 14,45 & 15,74 & 0,92 \\
\hline 4 & 90 & 18,23 & 18,51 & 18,51 & - \\
\hline Média & & $12,89 \mathrm{a}$ & $12,41 \mathrm{~b}$ & $13,12 \mathrm{c}$ & $0,92 \mathrm{~d}$ \\
\hline 5 & 40 & 10,04 & 10,04 & 10,16 & - \\
\hline 5 & 50 & 11,62 & 11,00 & 11,47 & 0,96 \\
\hline 5 & 60 & 13,21 & 11,88 & 13,01 & 0,91 \\
\hline 5 & 70 & 15,44 & 13,67 & 15,02 & 0,91 \\
\hline 5 & 80 & 18,04 & 16,72 & 17,57 & 0,95 \\
\hline 5 & 90 & 23,36 & 22,28 & 22,28 & - \\
\hline \multirow[t]{2}{*}{ Média } & & $15,29 \mathrm{a}$ & $14,27 \mathrm{~b}$ & $14,92 \mathrm{c}$ & $0,93 \mathrm{~d}$ \\
\hline & \multicolumn{5}{|c|}{ Aglomerado } \\
\hline 6 & 40 & 8,57 & 8,57 & 8,60 & \\
\hline 6 & 50 & 10,01 & 9,52 & 10,08 & 0,94 \\
\hline 6 & 60 & 11,26 & 10,53 & 11,26 & 0,94 \\
\hline 6 & 70 & 13,16 & 11,78 & 13,06 & 0,90 \\
\hline 6 & 80 & 16,62 & 14,05 & 15,36 & 0,91 \\
\hline 6 & 90 & 17,56 & 17,98 & 17,98 & - \\
\hline Média & & $12,86 \mathrm{a}$ & $12,07 \mathrm{~b}$ & $12,72 \mathrm{c}$ & $0,92 \mathrm{~d}$ \\
\hline 7 & 40 & 8,74 & 8,74 & 8,62 & - \\
\hline 7 & 50 & 10,17 & 9,47 & 10,01 & 0,95 \\
\hline 7 & 60 & 11,41 & 10,41 & 11,38 & 0,91 \\
\hline 7 & 70 & 13,31 & 11,83 & 13,22 & 0,89 \\
\hline 7 & 80 & 15,43 & 13,86 & 15,28 & 0,91 \\
\hline
\end{tabular}

- - - - - - - - - - - - - - - Continued...
Tabela 3 - Cont.

Table 3-Cont.

\begin{tabular}{|c|c|c|c|c|c|}
\hline TRAT & UR & UE1 & UE2 & UE3 & HISTERESE \\
\hline $\begin{array}{c}7 \\
\text { Média } \\
\end{array}$ & 90 & $\begin{array}{c}17,94 \\
12,83 \mathrm{a} \\
\end{array}$ & $\begin{array}{c}17,61 \\
11,99 \mathrm{~b}\end{array}$ & $\begin{array}{c}17,61 \\
12,69 \mathrm{c} \\
\end{array}$ & $\overline{-}, 92 \mathrm{~d}$ \\
\hline 8 & 40 & 7,77 & 7,77 & 8,15 & - \\
\hline 8 & 50 & 9,40 & 8,70 & 9,36 & 0,93 \\
\hline 8 & 60 & 10,95 & 9,84 & 10,81 & 0,91 \\
\hline 8 & 70 & 12,57 & 10,78 & 12,50 & 0,86 \\
\hline 8 & 80 & 14,65 & 13,20 & 14,51 & 0,91 \\
\hline 8 & 90 & 17,27 & 16,82 & 16,82 & - \\
\hline Média & & $12,10 \mathrm{a}$ & $11,19 \mathrm{~b}$ & $12,03 \mathrm{c}$ & $0,90 \mathrm{~d}$ \\
\hline 9 & 40 & 8,31 & 8,31 & 8,44 & - \\
\hline 9 & 50 & 9,65 & 10,02 & 10,24 & 0,98 \\
\hline 9 & 60 & 11,05 & 10,93 & 11,14 & 0,98 \\
\hline 9 & 70 & 12,89 & 12,02 & 12,93 & 0,93 \\
\hline 9 & 80 & 15,13 & 14,39 & 15,09 & 0,95 \\
\hline 9 & 90 & 17,51 & 18,40 & 18,40 & - \\
\hline Média & & $12,42 \mathrm{a}$ & $12,34 \mathrm{~b}$ & $12,71 \mathrm{c}$ & $0,96 \mathrm{~d}$ \\
\hline 10 & 40 & 8,64 & 8,64 & 8,46 & - \\
\hline 10 & 50 & 9,89 & 9,53 & 10,04 & 0,95 \\
\hline 10 & 60 & 11,43 & 10,57 & 11,39 & 0,93 \\
\hline 10 & 70 & 13,28 & 11,53 & 13,28 & 0,87 \\
\hline 10 & 80 & 15,61 & 14,07 & 15,43 & 0,91 \\
\hline 10 & 90 & 17,86 & 18,03 & 18,03 & - \\
\hline Média & & $12,78 \mathrm{a}$ & $12,06 \mathrm{~b}$ & $12,77 \mathrm{c}$ & $0,91 \mathrm{~d}$ \\
\hline 11 & 40 & 8,32 & 8,32 & 8,46 & - \\
\hline 11 & 50 & 9,63 & 9,05 & 9,49 & 0,95 \\
\hline 11 & 60 & 10,80 & 10,22 & 10,66 & 0,96 \\
\hline 11 & 70 & 12,41 & 11,24 & 12,56 & 0,89 \\
\hline 11 & 80 & 14,75 & 13,43 & 14,31 & 0,94 \\
\hline 11 & 90 & 16,36 & 17,07 & 17,07 & - \\
\hline Média & & $12,04 \mathrm{a}$ & $11,55 \mathrm{~b}$ & $12,09 \mathrm{c}$ & $0,94 \mathrm{~d}$ \\
\hline \multicolumn{6}{|c|}{ Chapa Dura } \\
\hline 12 & 40 & 6,31 & 6,31 & 7,02 & - \\
\hline 12 & 50 & 7,94 & 7,12 & 7,43 & 0,96 \\
\hline 12 & 60 & 9,36 & 7,63 & 9,36 & 0,82 \\
\hline 12 & 70 & 10,68 & 8,85 & 10,48 & 0,84 \\
\hline 12 & 80 & 12,11 & 10,99 & 11,80 & 0,93 \\
\hline 12 & 90 & 14,45 & 13,53 & 13,53 & - \\
\hline Média & & $10,14 \mathrm{a}$ & $9,07 \mathrm{~b}$ & $9,94 \mathrm{c}$ & $0,89 \mathrm{~d}$ \\
\hline 13 & 40 & 7,13 & 7,13 & 8,08 & - \\
\hline 13 & 50 & 8,62 & 7,76 & 8,62 & 0,90 \\
\hline 13 & 60 & 9,46 & 8,62 & 9,78 & 0,88 \\
\hline 13 & 70 & 11,70 & 9,68 & 11,59 & 0,84 \\
\hline 13 & 80 & 12,98 & 11,91 & 13,19 & 0,90 \\
\hline 13 & 90 & 15,53 & 15,00 & 15,00 & - \\
\hline Média & & $10,90 \mathrm{a}$ & $10,02 \mathrm{~b}$ & $11,04 \mathrm{c}$ & $0,88 \mathrm{~d}$ \\
\hline 14 & 40 & 7,04 & 7,04 & 7,51 & - \\
\hline 14 & 50 & 8,43 & 7,88 & 8,71 & 0,90 \\
\hline 14 & 60 & 9,82 & 8,44 & 9,73 & 0,87 \\
\hline 14 & 70 & 11,21 & 9,55 & 11,40 & 0,84 \\
\hline 14 & 80 & 12,60 & 11,40 & 12,69 & 0,90 \\
\hline
\end{tabular}


Tabela 3 - Cont.

Table 3 - Cont.

\begin{tabular}{|c|c|c|c|c|c|}
\hline TRAT & UR & UE1 & UE2 & UE3 & HISTERESE \\
\hline 14 & 90 & 13,99 & 14,73 & 14,73 & _ \\
\hline \multirow[t]{2}{*}{ Média } & & $10,52 \mathrm{a}$ & $9,84 \mathrm{~b}$ & $10,80 \mathrm{c}$ & $0,88 \mathrm{~d}$ \\
\hline & & & MDF & & \\
\hline 15 & 40 & 8,10 & 8,10 & 7,98 & - \\
\hline 15 & 50 & 9,39 & 8,89 & 9,33 & 0,95 \\
\hline 15 & 60 & 10,29 & 9,77 & 10,35 & 0,94 \\
\hline 15 & 70 & 12,11 & 10,77 & 12,03 & 0,90 \\
\hline 15 & 80 & 14,14 & 12,94 & 14,14 & 0,91 \\
\hline 15 & 90 & 17,13 & 17,22 & 17,22 & - \\
\hline Média & & $11,86 \mathrm{a}$ & $11,28 \mathrm{~b}$ & $11,84 \mathrm{c}$ & $0,93 \mathrm{~d}$ \\
\hline 16 & 40 & 8,03 & 8,03 & 8,03 & - \\
\hline 16 & 50 & 9,32 & 8,86 & 9,23 & 0,96 \\
\hline 16 & 60 & 10,30 & 9,75 & 10,36 & 0,94 \\
\hline 16 & 70 & 12,05 & 10,73 & 12,05 & 0,89 \\
\hline 16 & 80 & 14,07 & 12,92 & 14,04 & 0,92 \\
\hline 16 & 90 & 17,06 & 16,89 & 16,89 & - \\
\hline Média & & $11,80 \mathrm{a}$ & $11,20 \mathrm{~b}$ & $11,77 \mathrm{c}$ & $0,93 \mathrm{~d}$ \\
\hline 17 & 40 & 7,18 & 7,18 & 6,66 & - \\
\hline 17 & 50 & 8,62 & 7,97 & 8,10 & 0,98 \\
\hline 17 & 60 & 9,01 & 9,15 & 9,27 & 0,99 \\
\hline 17 & 70 & 10,58 & 9,53 & 10,70 & 0,89 \\
\hline 17 & 80 & 12,02 & 11,75 & 12,41 & 0,95 \\
\hline 17 & 90 & 15,54 & 16,06 & 16,06 & - \\
\hline \multirow[t]{2}{*}{ Média } & & $10,49 \mathrm{a}$ & $10,27 \mathrm{~b}$ & $10,47 \mathrm{c}$ & $0,96 \mathrm{~d}$ \\
\hline & & & OSB & & \\
\hline 18 & 40 & 8,02 & 8,02 & 7,96 & - \\
\hline 18 & 50 & 10,45 & 9,06 & 10,43 & 0,87 \\
\hline 18 & 60 & 10,68 & 9,90 & 10,73 & 0,92 \\
\hline 18 & 70 & 12,58 & 11,16 & 12,36 & 0,90 \\
\hline 18 & 80 & 14,51 & 13,42 & 14,90 & 0,90 \\
\hline 18 & 90 & 18,84 & 16,82 & 16,82 & - \\
\hline Média & & $12,51 \mathrm{a}$ & $11,40 \mathrm{~b}$ & $12,20 \mathrm{c}$ & $0,90 \mathrm{~d}$ \\
\hline 19 & 40 & 8,58 & 8,58 & 8,37 & - \\
\hline 19 & 50 & 10,00 & 9,44 & 9,97 & 0,95 \\
\hline 19 & 60 & 11,53 & 10,50 & 11,45 & 0,92 \\
\hline 19 & 70 & 13,38 & 11,75 & 13,25 & 0,89 \\
\hline 19 & 80 & 15,37 & 14,14 & 15,16 & 0,93 \\
\hline 19 & 90 & 18,05 & 15,02 & 15,02 & - \\
\hline Média & & 12,82 a & $11,57 \mathrm{~b}$ & $12,20 \mathrm{c}$ & $0,92 \mathrm{~d}$ \\
\hline 20 & 40 & 8,08 & 8,08 & 8,32 & - \\
\hline 20 & 50 & 9,65 & 9,08 & 9,90 & 0,92 \\
\hline 20 & 60 & 11,01 & 10,15 & 11,03 & 0,92 \\
\hline 20 & 70 & 12,77 & 11,30 & 12,92 & 0,87 \\
\hline 20 & 80 & 15,01 & 13,69 & 15,13 & 0,90 \\
\hline 20 & 90 & 19,72 & 18,09 & 18,09 & - \\
\hline \multirow[t]{2}{*}{ Média } & & $12,71 \mathrm{a}$ & $11,73 \mathrm{~b}$ & $12,56 \mathrm{c}$ & $0,90 \mathrm{~d}$ \\
\hline & \multicolumn{5}{|c|}{ Madeira cimento } \\
\hline 21 & 40 & 8,15 & 8,15 & 8,56 & - \\
\hline 21 & 50 & 9,09 & 8,47 & 9,19 & 0,92 \\
\hline 21 & 60 & 9,71 & 8,83 & 9,85 & 0,90 \\
\hline
\end{tabular}

Tabela 3 - Cont.

Table 3 - Cont.

\begin{tabular}{cccccc}
\hline TRAT & UR & UE1 & UE2 & UE3 & HISTERESE \\
\hline 21 & 70 & 10,39 & 9,15 & 10,50 & 0,87 \\
21 & 80 & 11,38 & 10,10 & 11,41 & 0,88 \\
21 & 90 & 13,56 & 12,57 & 12,57 & - \\
Média & \multicolumn{7}{c}{$10,38 \mathrm{a}$} & $9,54 \mathrm{~b}$ & $10,35 \mathrm{c}$ & $0,89 \mathrm{~d}$ \\
\hline 22 & 40 & 8,97 & 8,97 & 8,83 & - \\
22 & 50 & 9,84 & 9,35 & 9,60 & 0,97 \\
22 & 60 & 10,37 & 9,69 & 10,11 & 0,96 \\
22 & 70 & 11,06 & 9,96 & 10,84 & 0,92 \\
22 & 80 & 11,93 & 10,88 & 11,54 & 0,94 \\
22 & 90 & 14,68 & 12,79 & 12,79 & - \\
Média & $11,14 \mathrm{a}$ & $10,27 \mathrm{~b}$ & $10,62 \mathrm{c}$ & $0,95 \mathrm{~d}$ \\
\hline \multicolumn{5}{c}{ Madeira plástico } \\
\hline 23 & 40 & 5,18 & 5,18 & 5,53 \\
23 & 50 & 6,29 & 6,04 & 6,49 & - \\
23 & 60 & 7,36 & 6,65 & 7,41 & 0,93 \\
23 & 70 & 8,17 & 7,31 & 8,22 & 0,89 \\
23 & 80 & 10,30 & 8,98 & 10,20 & 0,88 \\
23 & 90 & 12,57 & 11,47 & 11,47 & - \\
Média & $8,31 \mathrm{a}$ & $7,60 \mathrm{~b}$ & $8,22 \mathrm{c}$ & $0,90 \mathrm{~d}$ \\
\hline \multicolumn{5}{c}{$\mathrm{HDF}$} \\
\hline 24 & 40 & 7,78 & 7,78 & 7,84 & - \\
24 & 50 & 9,23 & 8,79 & 9,29 & 0,95 \\
24 & 60 & 10,22 & 10,01 & 10,35 & 0,97 \\
24 & 70 & 11,67 & 10,42 & 11,74 & 0,89 \\
24 & 80 & 13,68 & 12,30 & 13,56 & 0,91 \\
24 & 90 & 18,77 & 15,94 & 15,94 & - \\
Média & $11,89 \mathrm{a}$ & $10,87 \mathrm{~b}$ & $11,45 \mathrm{c}$ & $0,93 \mathrm{~d}$ \\
\hline
\end{tabular}

* Médias por coluna seguidas da mesma letra não diferenciam entre si, pelo teste de Scott-Knott a 5\% de probabilidade.

* Averages for column followed of same letter do not differentiate between itself $5 \%$ of probability, for the test of Scott-Knott.

Tabela 4 - Resumo da análise de variância da diferença entre os tratamentos

Table 4-Summary of the Analysis of variance for the difference among treatments

\begin{tabular}{lcc}
\hline FV & GL & QM \\
\hline Tratamento & 3 & $1,31803 * *$ \\
Erro & 20 & 0,15106 \\
\hline Total & 23 & \\
\hline
\end{tabular}

** Significativo a $1 \%$ de probabilidade, pelo teste $\mathrm{F}$.

** Significant $1 \%$ of probability, for test $F$.

Tabela 5 - Resumo da análise de variância da UE calculada e UE observada

Table 5 -Summary of the Analysis of variance for the UE found and UE observed

\begin{tabular}{lcc}
\hline \multicolumn{1}{c}{ FV } & GL & QM \\
\hline Tratamento & 7 & $9,5590 \mathrm{NS}$ \\
Erro & 40 & 11,8084 \\
\hline Total & 47 & \\
\hline
\end{tabular}

** Significativo a $1 \%$ de probabilidade, pelo teste $\mathrm{F}$.

** Significant $1 \%$ of probability, for test $F$. 
Tabela 6 - Valores médios da umidade de equilíbrio calculados pela Equação de Nelson e observados na câmara de climatização, na temperatura de $30{ }^{\circ} \mathrm{C}$

Table 6 - Mean values of equilibrium moisture content found by Nelson's equation and observed in the climatization chamber, at $30^{\circ} \mathrm{C}$

\begin{tabular}{|c|c|c|c|c|}
\hline & & & AGLOMERADO & \\
\hline UR & UECalculada (\%) & UE Observada (\%) & Diferença & Porcentagem (\%) \\
\hline 40 & 7,68 & 8,41 & 0,73 & 8,7 \\
\hline 50 & 8,92 & 9,68 & 0,76 & 7,9 \\
\hline 60 & 10,29 & 10,89 & 0,60 & 5,5 \\
\hline 70 & 11,89 & 12,47 & 0,58 & 4,7 \\
\hline 80 & 13,99 & 14,73 & 0,74 & 5,0 \\
\hline 90 & 17,34 & 17,57 & 0,23 & 1,3 \\
\hline Médias* & $11,68 \mathrm{a}$ & $12,37 \mathrm{a}$ & $0,61 \mathrm{~b}$ & \\
\hline & & & OSB & \\
\hline UR & UE Calculada (\%) & UE Observada (\%) & Diferença & Porcentagem $(\%)$ \\
\hline 40 & 7,09 & 8,22 & 1,13 & 13,7 \\
\hline 50 & 8,58 & 9,78 & 1,20 & 12,3 \\
\hline 60 & 10,20 & 10,78 & 0,57 & 5,3 \\
\hline 70 & 12,11 & 12,39 & 0,27 & 2,2 \\
\hline 80 & 14,61 & 14,59 & 0,01 & 0,1 \\
\hline 90 & 18,60 & 17,38 & 1,21 & 7,0 \\
\hline Médias & $11,86 \mathrm{a}$ & $12,19 \mathrm{a}$ & $0,73 \mathrm{~b}$ & \\
\hline & & & CHAPA DURA & \\
\hline UR & UE Calculada (\%) & UE Observada (\%) & Diferença & Porcentagem (\%) \\
\hline 40 & 5,13 & 7,06 & 1,93 & 27,3 \\
\hline 50 & 6,21 & 8,06 & 1,85 & 23,0 \\
\hline 60 & 7,38 & 9,13 & 1,75 & 19,2 \\
\hline 70 & 8,77 & 10,57 & 1,80 & 17,0 \\
\hline 80 & 10,58 & 12,19 & 1,60 & 13,1 \\
\hline 90 & 13,48 & 14,50 & 1,02 & 7,0 \\
\hline Médias & $8,59 \mathrm{a}$ & $10,25 \mathrm{a}$ & $1,66 \mathrm{c}$ & \\
\hline & & & MDF & \\
\hline UR & UE Calculada (\%) & UE Observada (\%) & Diferença & Porcentagem (\%) \\
\hline 40 & 6,36 & 7,70 & 1,34 & 17,4 \\
\hline 50 & 7,64 & 8,86 & 1,22 & 13,8 \\
\hline 60 & 9,03 & 9,81 & 0,77 & 7,8 \\
\hline 70 & 10,68 & 11,17 & 0,49 & 4,4 \\
\hline 80 & 12,83 & 13,11 & 0,29 & 2,2 \\
\hline 90 & 16,26 & 16,67 & 0,41 & 2,5 \\
\hline Médias & $10,47 \mathrm{a}$ & $11,22 \mathrm{a}$ & $0,75 \mathrm{~b}$ & \\
\hline
\end{tabular}

* Médias seguidas de mesma letra na linha (UE) e na coluna (diferença) não diferenciam entre si, pelo teste de Scott-Knott a 5\% de probabilidade. * Followed averages of same letter in the line (UE) and the column (difference), do not differentiate between itself $5 \%$ of probability, for the test of Scott-Knott.

Finalmente, com relação ao efeito do revestimento dos painéis na umidade de equilíbrio, ainda que não se tenha constatado estatisticamente alguma diferença significativa,

A

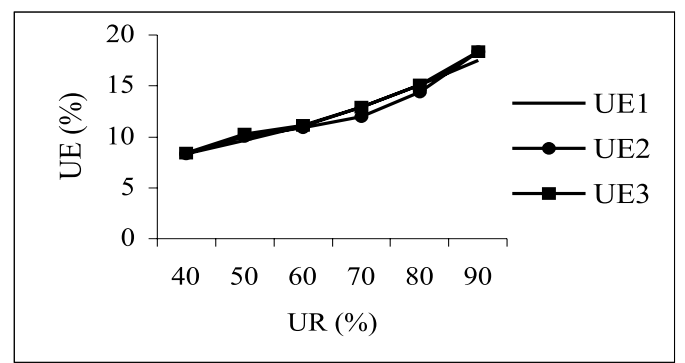

observou-se, no entanto, uma tendência de redução da relação A/D (histerese), aumentando, assim, a estabilidade dimensional dos painéis, conforme ilustrado na Figura 1.

B

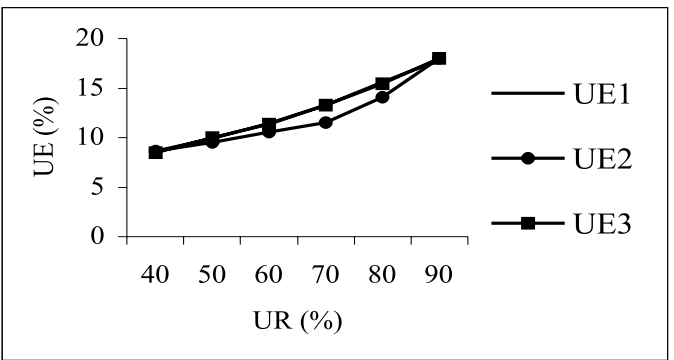

Figura 1 - Curvas de primeira dessorção (UE1), adsorção (UE2) e segunda dessorção (UE3) para o aglomerado com revestimento (A) e sem revestimento (B).

Figure 1 - Curves of first desorption (UE1), adsorption (UE2) and second desorption (UE3) for particleboard with coating $(A)$ and without coating (B). 


\section{CONCLUSÕES}

Os resultados permitiram as seguintes conclusões:

$\checkmark$ A espessura e o tipo de revestimento não influenciaram o coeficiente de histerese e a umidade de equilíbrio dos painéis.

$\checkmark$ Os valores obtidos através da equação de Nelson (1983) mostraram-se muito próximos daqueles observados na câmara de climatização.

$\checkmark$ A equação de Nelson (1983) se mostrou eficiente na estimativa da umidade de equilíbrio.

$\checkmark$ Não existe, em média, diferença de histerese entre os produtos estudados, indicando que todos apresentam a mesma estabilidade dimensional.

\section{SUGESTÕES}

Em trabalhos futuros sobre o assunto aqui abordado pode-se propor o seguinte:

$\checkmark$ É necessário desenvolver equações adequadas às condições climáticas brasileiras .

$\checkmark$ É preciso realizar um mapeamento nacional dos teores de umidade de equilíbrio de painéis de madeira, semelhante ao executado por Martins et al. (2003) sobre madeira maciça, visando orientar e apoiar o setor madeireiro em busca da melhor utilização da madeira e produtos dela derivados.

\section{REFERÊNCIAS BIBLIOGRÁFICAS}

ASSOCIAÇÃO BRASILEIRA DA INDÚSTRIA DE PAINÉIS DE MADEIRA - ABIPA. Relatório setorial. São Paulo: 2000. 25 p.

BURCH, D. M.; THOMAS, W. C.; FANNEY, A. H. Water vapor permeability measurements of common building materials. ASHRAE Transactions, v, 98, p. 486-494, 1992.
DURLO, M. A. Relação água-madeira. In: SEMINÁRIO SOBRE SECAGEM DE MADEIRA, 1 ., 1992, Santa Maria. Anais... Santa Maria: UFSM, CEPEF/FATEC, 1992. 100 p.

GALVÃO, A. P. M.; JANKOWSKY, I. P.

Secagem racional da madeira. São Paulo: Nobel, 1985. 112 p.

HEEBINK, D. M.; HASKELL, H. H. Effect of heat and humidity on the properties of high pressure laminates. Forest Products Journal, v. 12, n. 11, p. $542-548,1962$.

MARTINS, V.A. et al. Umidade de equilíbrio e risco de apodrecimento da madeira em condições de serviço no Brasil. Revista Brasil

Florestal, v. 76, p. 29 -34, 2003.

MESQUITA, J. B. Relação água-madeira. Lavras: 2000. (Notas de aula). (Material não publicado).

NELSON, R. M. A model for sorption of water vapor by cellulosic materials. Wood Fiber Science, v. 15, n. 1, p. 8-22, 1983.

SUCHSLAND, O. Linear higroscopic expansion of selected commercial particleboards. Forest Products Journal, v. 22, n. 11, 28-32, 1972.

WU, Q. Apllication of Nelson's sorption isotherm to wood composites and overlays. Wood Fiber Science, v. 3, n. 2, 187-191, 1999.

WU, Q.; SUCHSLAND, O. Prediction of moisture content and moisture content gradient of particleboard. Wood and Fiber Science, v.28, n. 2, p. 227-239, 1996. 